\title{
A Fuzzy Control for Optimizing the Design of Passive Electrical Circuits
}

\author{
Pedro A. Miranda-Romagnoli \\ Department of Mathematics and Physics \\ Autonomous University of Hidalgo \\ Pachuca, Mexico \\ Email:pmir@ieee.org
}

\author{
Norberto Hernández-Romero \\ Juan C. Seck-Tuoh-Mora \\ Department of Industrial Engineering \\ Autonomous University of Hidalgo \\ Pachuca, Mexico
}

\begin{abstract}
This paper exposes a fuzzy control to determine independent parameters of a passive circuit in order to obtain a desired output established by the design requirements. This is achieved using a vector representation of the circuit and varying the independent parameters by a fuzzy control based on three inference rules according to the output voltage. An analysis about the behavior of the control is explained and some examples of this procedure are depicted.
\end{abstract}

Index Terms-Computer aided design; fuzzy control; passive circuits.

\section{INTRODUCTION}

The automatic design of electrical circuits has been widely investigated due to the development of a huge set of practical applications; its progress can be classified in several ways with diverse interrelations among them. One tendency closer to electronics theory is using a modified representation of the circuit, as the modified nodal approach [1] taking advantage of the simplicity in nodal analysis. The circuit is decomposed into independent subnets which are studied using solutions from tensor analysis known as diakoptics. A different approach based in an optimal control formulation has been done in [2].

An additional direction is to employ algorithms from numerical analysis for obtaining an optimization exploiting computational resources. Many numerical methods have been developed for calculating both restricted and unrestricted results, firstly they were implemented for optimizing production, time and space in the design of circuits [3]. However, regardless of good benefits, these methods involve a deep comprehension about the mathematical properties of electrical circuits, complicating their implementation and application.

In this sense the aim of this paper is to propose an emergent approach for achieving a more accessible way to perform and find appropriated approximations in a short time for the optimization of electrical circuits, in particular by means of a fuzzy control. In an excited passive electrical circuit, there are independent parameters which must hold certain design requirements, in particular with a desired output voltage. A fuzzy control may adjust these parameters measuring the output voltage and applying inference rules based on the premise that a raising of the parameters increases the voltage in the output and vice versa.
In this way we balance the independent parameters up to the design requirements are accomplished. This manuscript takes only passive electrical circuits which are relevant in applications of coupling networks; in further works other types of electrical circuits will be studied. The paper is organized as follows: section II provides the fundaments of automatic design in electrical circuits, section III gives the concepts used for implementing a fuzzy control for optimizing the automatic design. Section IV depicts some examples of the procedure explained in the work and section $\mathrm{V}$ presents the concluding remarks of the paper.

\section{Automatic Design of ElectricAl CiRCUits}

The process of automatic design of electrical circuits is composed by two main parts; the first is the modeling and estimation of the solution describing the system. In this step there are a number of methods for solving particular cases; for instance, in a non-linear problem, Newton-Rapson algorithm is a classic tool. This one implies a high computational cost for large circuits, because a Jacobian must be evaluated in every integration step, although commonly few iterations are needed. The second part is the optimization procedure to reach the desired objective requirements; for this task, a recurrent tool is the utilization of first-order methods as the gradient one; Newton-family procedures, or a combination of both.

In this sense, the goal of the paper is to implement a different approach for the later case, looking for a simplification in the optimization of the model. For this reason a convenient matrix representation of electrical circuits is defined; the set of nodal equations representing an electrical circuit can be formulated as:

$$
g_{j}(\mathbf{x})=0 ; \quad j=1, \ldots, n
$$

where $g_{j}$ is the $j$-th nodal equation. Vector $\mathbf{x} \equiv$ $\left[\begin{array}{llllll}x_{1} & \cdots & x_{k} & x_{k+1} & \cdots & x_{k+n}\end{array}\right]^{T}$ contains $k$ independent parameters conformed by the admittances generated by passive elements such as resistances, capacitors and inductors; and $n$ dependent parameters specified by nodal voltages.

This formulation allows to pose the design problem as the minimization of a function $f$ for obtaining a desired condition over some of the voltage nodes: 


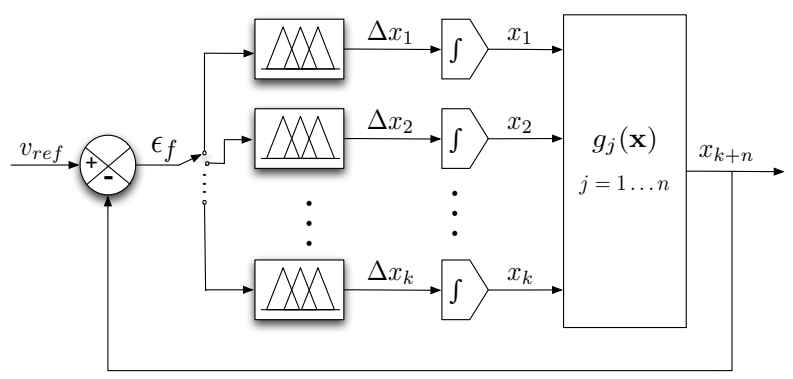

Fig. 1. Fuzzy control for optimizing the circuit design.

$$
\begin{array}{ll}
\operatorname{minimize}\left\{f(\mathbf{x}) \mid \mathbf{x} \in \mathbb{R}^{n}\right\} \\
\text { Subject to: } & g_{j}(\mathbf{x})=0 \\
& x_{i} \geq 0 \quad i=1, \ldots, m
\end{array}
$$

where $f: \mathbb{R}^{n} \rightarrow \mathbb{R}$. There are $m \leq k$ admittances generated by same number of resistances whose values are restricted to be positive. We emphasize the fact that the model of the circuit given in Eq. 1 must be solve at each numerical step, including Eq. 2 as restriction. An interesting case is to obtain a desired output voltage $v_{r e f}$ where the expression to reach (the objective function) may be presented as:

$$
f\left(x_{k+n}\right)=v_{r e f}-x_{k+n}
$$

In this way, we can define an error $\epsilon_{f}=f\left(x_{k+n}\right)$ where values near to 0 assure that the design is approximated to the required objective.

\section{FUZZY CONTROL SCHEME}

Fuzzy control has been widely developed and applied in different fields for optimizing and controlling several dynamical systems in engineering process control, pattern recognition, image processing, industrial reliability among others [4], [5].

In order to optimize the design of a given electrical circuit for obtaining a desired output voltage, this paper describes a methodology based on applying an inference system for adjusting each of the independent parameters taking as reference the difference between the actual output and the desired one. This approach has been explored before by other authors for designing specific electrical circuits, nevertheless there is not a general methodology which can be applied at least for a particular set of electrical circuits [6], [7]. The advantage of using a fuzzy approximation is that a deep mathematical characterization and knowledge of the circuit is not needed, contrary to the case of the most common numerical methods used for optimizing a given design.

The original part of the proposed configuration is that the whole control is in fact a set of switched fuzzy controls applied to the independent parameters of the electrical circuit. Each fuzzy control follows the Mamdani method (fuzzification, inference system, defuzzification); where the inference systems

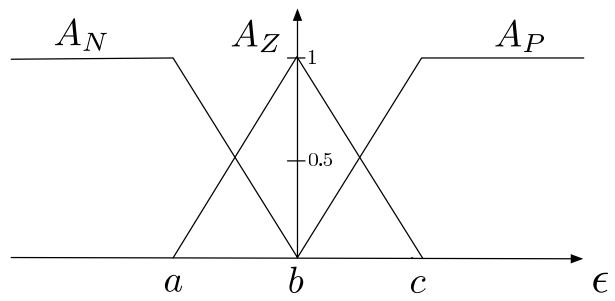

Fig. 2. Membership functions for the fuzzification of $\epsilon_{f}$.

are based on the experience in solving the optimization of an electrical circuit.

Let $A=\left\{U, \mu_{A}\right\}$ be a fuzzy set defined by a universe of discourse $U$ and a membership function $\mu: U \rightarrow[0,1]$. In our case, the universe of discourse is $\mathbb{R}$ and the control will be composed by a family of $k$ fuzzy inference systems, one for each independent parameter (Fig. 1).

Every inference system has as the entry $\epsilon_{f}$ obtained by Eq. 3. All the inference systems are implemented taking the three fuzzy sets showed in Fig. 2. The membership functions for the previous sets are defined in Equation 4.

$$
\begin{aligned}
& A_{N}=\left\{(-\infty, b], \mu_{N}\right\} \\
& A_{Z}=\left\{[a, c], \mu_{Z}\right\} \\
& A_{P}=\left\{[b, \infty), \mu_{P}\right\}
\end{aligned}
$$

The control realize a fuzzification of $\epsilon_{f}$ for obtaining a crisp control action which shall be serially applied for updating every $x_{i} \in \mathbf{x}$, therefore $k$ complete loops of the control are needed for actualizing all the elements in $\mathbf{x}$. On the other way, a decrement in the impedance generates a reduction in the nodal voltage of the element. In Eq. 3, if $\epsilon_{f} \neq 0$ then $x_{n+k} \neq v_{\text {ref }}$ and the inference system is implemented for each $x_{i}$ as follows:

$$
\begin{array}{llllllll}
I F & \epsilon_{f} & \text { is } & A_{N} & \text { THEN } & \delta_{N} & \text { is } & B_{N} \\
I F & \epsilon_{f} & \text { is } & A_{Z} & \text { THEN } & \delta_{Z} & \text { is } & B_{Z} \\
I F & \epsilon_{f} & \text { is } & A_{P} & \text { THEN } & \delta_{P} & \text { is } & B_{P}
\end{array}
$$

where for $J \in\{N, Z, P\}, \delta_{J}$ is the linguistic control action and $B_{J}$ is the singlenton corresponding to $A_{J}$ (Figure 3 ).

In the defuzzification step, the control action described in a linguistic form will be transformed in a crisp value defining a new value for $\Delta x_{i}$ by means of calculating the centroid over singleton membership functions.

\section{ILlustrative EXAMPLES}

This section presents two examples of passive circuits, where the second one includes two non-linear elements. In order to solve them, the parameters defining the input membership functions in the universe of discourse are specified as: $a=-1, b=0$ and $c=1$. These values were selected based on the practical experience in optimizing this kind of circuits. 


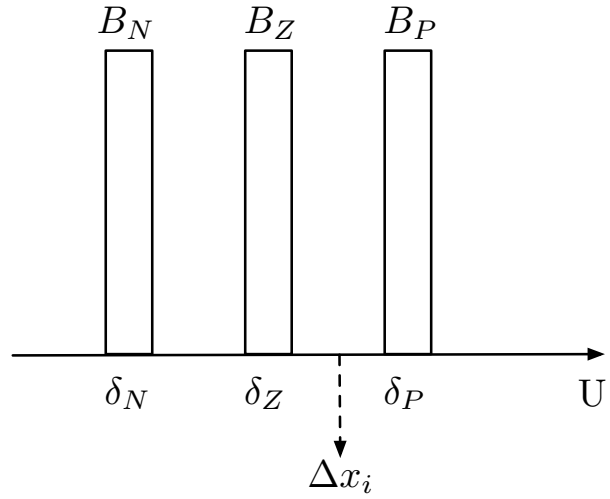

Fig. 3. Singletons for the defuzzification of $\Delta x_{i}$.

Example 1. The following example illustrates the design of a passive circuit with both independent and dependent parameters, in particular we have taken a circuit with two nodes showed in Figure 4.

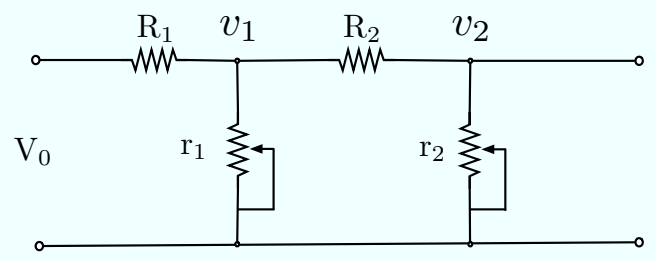

Fig. 4. Passive circuit with two nodes.

This circuit has two admittances as independent parameters; given by $y_{1}=\frac{1}{r_{1}}, y_{2}=\frac{1}{r_{2}}$ which are defined in the first two elements in $\mathbf{x}$ as $x_{1} \equiv y_{1}, x_{2} \equiv y_{2}$. The symbols of variable elements for $r_{1}$ y $r_{2}$ describe that their values are adjusted by means of the fuzzy control, up to reach the desired output $v_{2}$ stipulated in the objective function by $v_{\text {ref }}$. On the other hand, the resistances $R_{1}$ and $R_{2}$ have values fixed at the beginning of the process; there are two dependent parameters in this example defined by the nodal voltages, complementing the vector $\mathbf{x}$ by the equivalences $x_{3} \equiv v_{1}$ and $x_{4} \equiv v_{2}$.

TABLE I

RESULTS TAKING DISTINCT INITIAL VALUES WITH $\epsilon=1 \times 10^{-9}$.

\begin{tabular}{|c|ccc|c|}
\hline Initial Guess & $r_{1}(\Omega)$ & $r_{2}(\Omega)$ & $v_{2}(V)$ & \# of Iterations \\
\hline$x_{i}=0, \forall i$ & 0.383 & 0.721 & 0.099 & 107 \\
$x_{i}=1, \forall i$ & 0.406 & 0.683 & 0.100 & 101 \\
\hline
\end{tabular}

Table I presents the design results for the circuit in Fig. 4 taking $v_{\text {ref }}=0.1 \mathrm{~V}$, for two different initial values in $x$ and an error $\epsilon=1 \times 10^{-9}$, the values are truncated in three decimal places to simplify their presentation.

In both runs we have a similar number of iterations; a relevant point is that distinct solutions are obtained according to the selected initial conditions. Fig. 5(a) and (b) show the dynamics of the fuzzy control when all the parameters are equal to one and zero respectively in the start point.

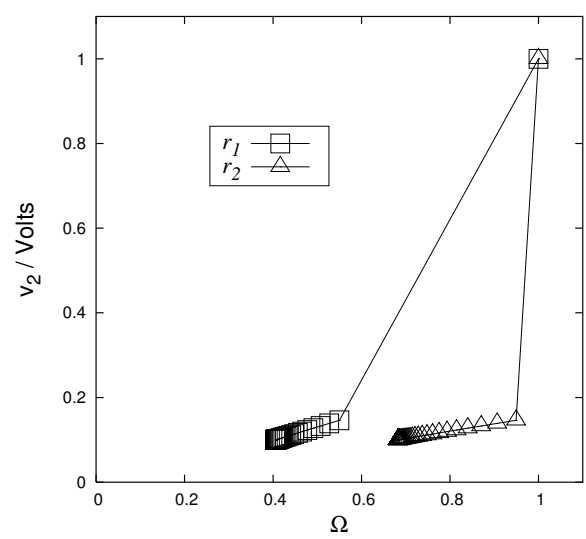

(a) Initial values equal to one.

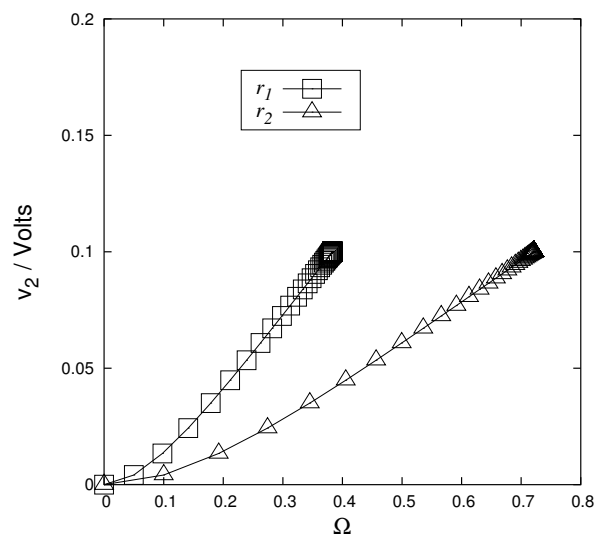

(b) Initial values equal to zero.

Fig. 5. Dynamics of $r_{1}$ and $r_{2}$ provided by the fuzzy control.

Example 2. Fig. 6 shows a circuit with three nodes and two non-linear dependent admittances $H_{i}=c_{1}+c_{2}\left(v_{i}-v_{i+1}\right)$, where $i \in\{1,2\}$ and $c_{1}, c_{2}$ are constants fixed at the beginning of the process.

In this way we have three independent parameters $y_{1}=\frac{1}{r_{1}}$, $y_{2}=\frac{1}{r_{2}}$ and $y_{3}=\frac{1}{r_{3}}$ and three dependent parameters $v_{1}, v_{2}, v_{3}$; thus the variable vector has six elements $\mathbf{x}=$ $\left[x_{1} \cdots x_{6}\right]^{T}$.

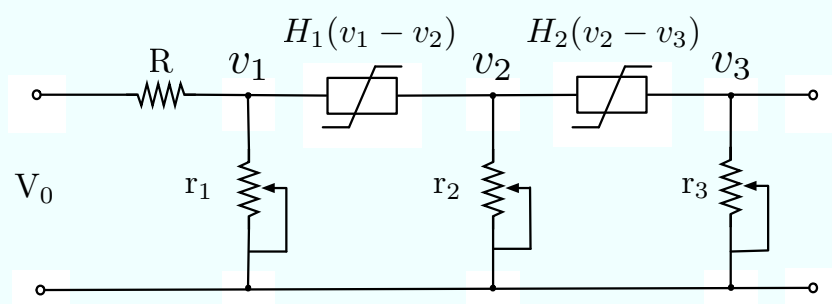

Fig. 6. Non-linear passive circuit.

The numerical results corresponding to distinct initial conditions when $v_{r e f}=0.3 \mathrm{~V}$ are described in Table II.

In the table we can observe that three different solutions are obtained and too-large initial values yield a longer number 
TABLE II

DESIGN RESULTS FOR DIVERSE INITIAL VALUES .

\begin{tabular}{|r|cccc|r|}
\hline $\begin{array}{r}\text { Initial Guess } \\
x_{i}, \forall i\end{array}$ & $r_{1}(\Omega)$ & $r_{2}(\Omega)$ & $r_{3}(\Omega)$ & $v_{3}(V)$ & $\begin{array}{r}\# \text { of } \\
\text { Iterations }\end{array}$ \\
\hline 0 & 2.161 & 2.059 & 1.989 & 0.300 & 95 \\
1 & 1.560 & 2.348 & 2.325 & 0.300 & 89 \\
10 & 1.697 & 2.090 & 2.370 & 0.300 & 99 \\
100 & 1.697 & 2.090 & 2.370 & 0.300 & 189 \\
\hline
\end{tabular}

of iteration for getting the desired design conditions.

\section{CONCLUDING REMARKS}

The proposed methodology based on fuzzy rules is simpler than the usually-sophisticated traditional techniques, such as newton's method, both in the mathematical treatment and in the computational implementation. On the other hand, execution times are similar for the examples described in the paper. In this way we have showed that a fuzzy control based on three membership functions in the input, three inference rules and three singlenton membership functions for the output is able to design passive circuits. In this case the inference system is straightforward but is not adequate for other types of problem, for instance, transistor-based circuits.

Further work is to use the results obtained in this paper for treating other type of more complicated circuits containing active elements. Another direction is to explore more complex membership functions for yielding better results in designing more general electrical circuits. Different approaches of emergent computing must be explored in this sense, as genetic algorithms and cellular automata with the aim of taking advantage of their simplicity and computational advantages for establishing new alternatives for solving problems which up to now are only resolved by complex numerical procedures.

\section{ACKNOWLEDGMENT}

Authors would like to thank system PROMEP and CONACYT CB-2007/83554 for supporting the present work.

\section{REFERENCES}

[1] C. Ho, A. E. Ruehli, and P. Brennan, "The modified nodal approach to network analysis," IEEE Trans. on Circuits and Syst., vol. CAS-22, pp. 504-509, 1975.

[2] A. Zemliak, E. Rios, P. Miranda, and K. Zemliak, "On time-optimal procedure for analog system design," Journal of Applied Research and Technology, vol. 2, no. 1, pp. 32-53, April 2004.

[3] R. K. Brayton, G. D. Hachtel, and A. L. Sangiovanni-Vicentelli, "A survay of optimisation techniques for integrated-circuit design," IEEE Proc., vol. 69, no. 10, pp. 1334-1362, 1981.

[4] K. Tanaka, A introduction to fuzzy logic for practical applications. Springer, 1997.

[5] T. J. Ross, J. Booker, and W. J. Parkinson, Fuzzy logic and probability applications: bridging the gap. SIAM, 2002.

[6] M. Fares and B. Kaminska, "Fpad: A fuzzy nonlinear programming approach to analog circuit design," IEEE Trans. on Computer-Aided Design, vol. 14, no. 7, pp. 785-793, july 1995.

[7] G. Oltean, C. Miron, and E.Mocean, "Multiobjective optimization method for analog circuits design based on fuzzy logic," in 9th. Intl. Conf. on Electronics, Circuits and Systems, vol. 2, september 2002, pp. 777-780. 